\title{
A TYPE OF NEAREST POINT SET IN A COMPLETE $l$-GROUP ${ }^{1}$
}

\author{
MICHAEL KEISLER
}

\begin{abstract}
A theorem by W. D. L. Appling (Riv. Mat. Univ. Parma, (3) 2 (1973), 251-276) demonstrates that a $C$-set is a nearest point set in ba( $S, \Sigma)$ with respect to the variation norm. This paper demonstrates an analogous result for a generalized form of $C$-set in a complete l-group with distance with respect to the norm being replaced by a stronger property definable in a complete l-group (distance between elements $x$ and $y$ of a complete $l$-group $G$ is taken to be $|x-y|)$. The result is then shown to be a characterization of sets possessing the stronger property in the case of a complete vector lattice, but not a characterization in the case of a complete l-group.
\end{abstract}

1. Introduction. There are obvious analogies between a norm on a space and the absolute value of elements of an l-group. Using $|x-y|$ as our concept of "distance", we will characterize the "nearest point" sets in a complete vector lattice, and demonstrate that the characterization fails in the case of a complete $l$-group. It is noted in [3, pp. 366, 371] that in a uniformly monotone Banach lattice (UMB-lattice) $\||x|\|=\|x\|$, and if $0 \leqslant x<y$, then $\|x\|<\|y\|$. Therefore, in a UMB-lattice, every nearest point set of the type described above is a nearest point set with respect to the norm. The converse is not true, however, as a closed circular disc in the plane with $\|(x, y)\|=|x|$ $+|y|$ readily demonstrates.

The general properties of l-groups, as presented in [3], are assumed throughout. Necessary properties not specifically proven in [3] are presented in the lemma below.

LEMMA 1. Let $G$ be a complete l-group and $\{a, b, c\} \subseteq G$,

(i) if $|a| \wedge|b|=0$, then $|a+b|=|a|+|b|$,

(ii) if $a \wedge b \wedge c \geqslant 0$, then $(a+b) \wedge c \leqslant a \wedge c+b \wedge c$,

(iii) ||$a|-| b|| \leqslant|a-b| \wedge|a+b|$.

Proof. The triangle inequality implies $|a| \leqslant|a-b|+|b|$ and $|b| \leqslant \mid a-$

Presented to the Society, January 23, 1975 under the title $A$ characterization of a type of nearest point set in complete l-groups; received by the editors June 18, 1976.

AMS (MOS) subject classifications (1970). Primary 06A60, 06A65, 26A51, 46A40; Secondary 28A10, 47B55.

Key words and phrases. Complete $l$-group, complete vector lattice, complex, $l$-nearest point set, normal subgroup, projection operator.

${ }^{1}$ This article contains, in part, research undertaken to fulfill requirements for the $\mathrm{Ph}$. $\mathrm{D}$. at North Texas State University, Denton, Texas, in 1974. 
$b|+| a \mid$, from which it follows that

$$
|| a|-| b||=(|a|-|b|) \vee(|b|-|a|)<|a-b| \text {. }
$$

Since $|b|=|-b|$, we have (iii). (ii) follows easily from the inequality $(a+b)$ $\wedge c<(a+b) \wedge(c+b) \wedge(a+c) \wedge(c+c)$. Finally, (i) can be proven by applying (iii);

$$
\begin{aligned}
|| a|-| b|| & \leqslant|a+b|<|a|+|b|=|a| \vee|b|-|a| \wedge|b| \\
& =(|a|-|b|) \vee 0+|b|+(-|a|) \vee(-|b|) \\
& =(|a|-|b|) \vee 0+(|b|-|a|) \vee 0=|| a|-| b|| .
\end{aligned}
$$

Definition. Let $G$ be an l-group. $S \subseteq G$ is an l-nearest point set (l-nps) iff for $x \in G$, there is $x^{\prime} \in S$ such that if $s \in S$ and $s \neq x^{\prime}$, then $\left|x-x^{\prime}\right|<\mid x$ - $s \mid$. (For an $l$-nps $S$, denote the nearest point map from $G$ to $S$ by $f_{S}$; i.e., for $x$ in $G$, the nearest point in $S$ is $f_{S}(x)$.)

Some basic properties of $l$-nearest point sets are given by the lemma below. Although the main results of this paper deal with complete l-groups, this lemma takes $G$ to be an arbitrary l-group.

LEMMA 2. Let $G$ be an l-group with l-nps $S$. For $x \in G, S-x$ is an l-nps (with $f_{S-x}(z)=f_{S}(z+x)-x$, for $z \in G$ ), $-S$ is an l-nps if $G$ is abelian (with $f_{-S}(z)=-f_{S}(-z)$, for $z$ in $G$ ), and if $S^{\prime} \subseteq S$ such that $x=\sup S^{\prime}$ exists, then $x \in S$.

Proof. For $z \in G$,

$$
\left|z-\left[f_{S}(z+x)-x\right]\right|=\left|z+x-f_{S}(z+x)\right|,
$$

and the latter is $\langle|z+x-s|=|z-(s-x)|$, for $s-x$ in $S-x$, unless $s-x=f_{S}(z+x)-x$; hence $S-x$ is an $l$-nps. For $z \in G, \mid z-$ $\left(-f_{S}(-z)\right)|=|(-z)-f_{S}(-z) \mid$, and the latter is less than $|(-z)-s|=\mid z-$ $(-s)$, for $-s \in-S$, unless $-s=-f_{S}(-z) ;-S$ is an $l$-nps. Finally, given $x=\sup S^{\prime}$,

$$
\begin{aligned}
\left|x-f_{S}(x)\right| & \leqslant \inf \left\{|x-s| \mid s \in S^{\prime}\right\}=\inf \left\{x-s \mid s \in S^{\prime}\right\} \\
& =x-\sup S^{\prime}
\end{aligned}
$$

hence $\left|x-f_{S}(x)\right|=0$ and $x=f_{S}(x) \in S$.

2. Some $l$-nearest point sets. The study of projection operators has a very long history. Bochner and Phillips noted in [4] that the function $p_{Y}(x)=$ $\sup \{x \wedge y \mid y \in Y\}$, for $x \geqslant 0$ and $Y$ a normal subspace of a complete vector lattice, provided a decomposition (Lebesgue decomposition for $\mathrm{ba}(S, \Sigma)$, where $Y$ is a principal normal subspace) of an element $x$ into $x-p_{Y}(x)$ and $p_{Y}(x)$ such that $\left|x-p_{Y}(x)\right| \wedge\left|p_{Y}(x)\right|=0$, where $p_{Y}$ has been extended to the entire vector lattice as follows; $p_{Y}(x)=p_{Y}\left(x^{+}\right)-p_{Y}\left(x^{-}\right)$, for $x$ a member of the vector lattice, with notation as in [4]. Kakutani also studied principal projection operators in [5], using the form $p_{y}(x)=\sup \{x \wedge n y \mid n=$ $1,2,3, \ldots\}$. Applying Lemma 1 , we have that 


$$
\left|x-p_{Y}(z)\right|=\left|x-p_{Y}(x)\right|+\left|p_{Y}(x-z)\right|>\left|x-p_{Y}(x)\right|,
$$

if $p_{Y}(z) \neq p_{Y}(x)$, since projection operators are additive and the singularity property mentioned above applies to $x-p_{Y}(x)$ and any member of $Y$. Hence every normal subspace is an l-nps. Appling [1] observed that the Lebesgue decomposition, using Kakutani's representation for the projection operator, provided a proof of a nearest point property in $\mathrm{ba}(S, \Sigma)$ with respect to the variation norm. As noted in the introduction, every $l$-nps in a UMB-lattice, which $\operatorname{ba}(S, \Sigma)$ with variation norm is, is a nearest point set, so the observation about normal subspaces above implies Appling's result.

Pinsker studied projection operators as well in [7] and [8] (the material contained herein is taken from a summary published later in English [6]). He established the properties of projection operators mentioned above for complete $l$-groups, but more to the point of this paper, he examined subsets of the positive cone called complexes.

Defintion. Let $G$ be a complete $l$-group with positive cone $P$. A subset $A$ of $P$ is a complex provided (1) $x \in A$ implies $[0, x] \subseteq A$ (where $[0, x]=\{y \mid 0$ $\leqslant y \leqslant x\}$ ), (2) $E \subseteq A$ and $E$ bounded implies sup $E \in A$, and (3) for any $x>0$, there is a positive integer $n$ such that $n x \notin A$.

Appling studied subsets of $\mathrm{ba}(S, \Sigma)$ which were, as we shall show, direct sums of sets of the form $A+(-A)$, where $A$ is a complex, and a normal subspace. He termed the subsets $C$-sets (see [2]); their definition is essentially the same as that of Pinsker for a complex except that (1) becomes $\left(1^{\prime}\right), x \in A$ implies $[-|x|,|x|] \subseteq A$, and (3) is omitted. The approach taken below owes much to that taken by Appling.

Henceforth $G$ is assumed to be a complete $l$-group.

Definition. $I=\{\alpha \mid \alpha: P \rightarrow P, \alpha \subseteq \geqslant, \alpha$ is isotone, idempotent and subadditive $\}$.

Lemma 3. If $\alpha \in I$ and $\{x, y\} \subseteq P$, then $y \leqslant \alpha(x)$ implies $y=\alpha(y)$.

Proof. $\alpha(x)=\alpha(\alpha(x)) \leqslant \alpha(\alpha(x)-y)+\alpha(y) \leqslant \alpha(x)-y+\alpha(y)$.

Lemma 4. If $x, y \in P$ and $\alpha \in I$, then $x \wedge \alpha(y)=\alpha(x \wedge y)=\alpha(x) \wedge y$.

PROOF. Since $\alpha$ is isotone and a subset of $\geqslant, \alpha(x \wedge y) \leqslant x \wedge \alpha(y) \leqslant x \wedge$ $y$. Hence $\alpha^{2}=\alpha$ implies $\alpha(x \wedge y) \leqslant \alpha(\alpha(x \wedge y)) \leqslant \alpha(x \wedge y)$. By Lemma 3, $\alpha(x \wedge \alpha(y))=x \wedge \alpha(y)$ and thus $\alpha(x \wedge y)=x \wedge \alpha(y)$. Similarly for $\alpha(x)$ $\wedge y$.

Lemma 5. $\alpha \in I$ iff $\alpha(x)=\sup \{x \wedge y \mid y \in S\}$, for some $S \subseteq P$.

PRoof. By Lemma 4, if $\alpha \in I$, then $\alpha(x \wedge \alpha(z))=x \wedge \alpha(z)$, for $x, z \in P$. Thus

$$
\alpha(x) \geqslant \sup \{x \wedge \alpha(z) \mid z \in P\} \geqslant x \wedge \alpha(x)=\alpha(x),
$$

and the theorem is proven for $S=\alpha(P)$. If $S \subseteq P$ and $\alpha(x)=\sup \{x \wedge y \mid y$ $\in S\}$, then clearly $\alpha \subseteq \geqslant$ and $\alpha$ is isotone. And 


$$
\begin{aligned}
\alpha(\alpha(x)) & =\sup \{\alpha(x) \wedge y \mid y \in S\} \\
& \geqslant \sup \{(x \wedge y) \wedge y \mid y \in S\}=\alpha(x) \geqslant \alpha(\alpha(x))
\end{aligned}
$$

implies $\alpha^{2}=\alpha$. Finally, Lemma 1(ii) implies $\alpha(x+y) \leqslant \alpha(x)+\alpha(y)$.

The last lemma allows us to note that both the notion of complex (by defining $\alpha_{A}(x)=\sup \{x \wedge a \mid a \in A\}$, for each complex $\left.A\right)$ and projection operator is included in the class $I$. If we define $(\alpha+\beta)(x)=\alpha(x)+\beta(x-$ $\alpha(x)),(\alpha \wedge \beta)(x)=\alpha(x) \wedge \beta(x)$ and $(\alpha \vee \beta)(x)=\alpha(x) \vee \beta(x)$, for $x \in$ $P$, and $\alpha \leqslant \beta$ iff $\alpha(x) \leqslant \beta(x)$, for $x \in P$, then,$+ \wedge$ and $\vee$ are binary operations on $I$ and $\leqslant$ is a partial order on $I$ compatible with + . Furthermore, $O(x)=0$, and $1(x)=x$, for $x \in P$, are lower and upper bounds in $I$.

If we use the notation $[-\alpha, \beta]=-\alpha(P)+\beta(P)$ (which, in turn, = $\left\{x \mid x^{+}=\beta\left(x^{+}\right)\right.$and $\left.\left.x^{-}=\alpha\left(x^{-}\right)\right\}\right)$, then we have a convenient way of describing a large class of $l$-nearest point sets. Intervals of the form $[-\alpha, \alpha]$, for $\alpha \in I$, are $C$-sets. Appling [2] showed that such intervals in ba $(S, \Sigma)$ form nearest point sets with respect to the variation norm. The result below extends Appling's to $l$-nearest point sets in a complete $l$-group.

THEOREM 1. Let $G$ be a complete l-group. If $\alpha, \beta \in I$, then $[-\alpha, \beta]$ is an l-nps, and hence $x+[-\alpha, \beta]$ is an l-nps, for $x \in G$.

Proof. For $x \in G$, let $f(x)=\beta\left(x^{+}\right)-\alpha\left(x^{-}\right)$, where $x^{+}=x \vee 0$ and $x^{-}=(-x) \bigvee 0$. Then for $x \in G$ and $z \in[-\alpha, \beta]$, such that $z \neq f(x)$, we know $\alpha\left(x^{-}\right) \neq z^{-}$or $\beta\left(x^{+}\right) \neq z^{+}$, from which it follows that

$$
\begin{aligned}
& |x-f(x)|=\left|\left(x^{+}-\beta\left(x^{+}\right)\right)-\left(x^{-}-\alpha\left(x^{-}\right)\right)\right| \\
& =x^{+}-\beta\left(x^{+}\right)+x^{-}-\alpha\left(x^{-}\right) \\
& <x^{+}-x^{+} \wedge z^{+}+z^{+}-x^{+} \wedge z^{+}+x^{-}-x^{-} \wedge z^{-}+z^{-}-x^{-} \wedge z^{-} \\
& =\mid\left(x^{+}-x^{+} \wedge z^{+}+z^{-}-x^{-} \wedge z^{-}\right) \\
& \quad-\left(x^{-}-x^{-} \wedge z^{-}+z^{+}-x^{+} \wedge z^{+}\right)|=| x-z \mid,
\end{aligned}
$$

by noting that

$$
\begin{aligned}
0 & =x^{+} \wedge x^{-}=z^{+} \wedge z^{-} \\
& =\left(x^{+}-x^{+} \wedge z^{+}\right) \wedge\left(z^{+--} x^{+} \wedge z^{+}\right) \\
& =\left(x^{-}-x^{-} \wedge z^{-}\right) \wedge\left(z^{-}-x^{-} \wedge z^{-}\right)
\end{aligned}
$$

and applying (i) and (ii) of Lemma 1.

For $x \in P$, let $\tau_{x}(z)=z \wedge x$, for $z \in P$, then $\tau_{x} \in I$. If $x, y \in P$, then $\left[-\tau_{x}, \tau_{y}\right]=[-x, y]$, using the usual interval notation for a partially ordered set, and we have the following.

Corollary. Let $G$ be a complete l-group. If $x, y \in G$, and $x \leqslant y$, then $[x, y]$ is an l-nps.

Not all $l$-nearest point sets in a complete $l$-group are necessarily of the form given in Theorem 1. For instance, the set of integers forms a complete 
$l$-group, and $\{0,3\}$ is an $l$-nps. Since $0<2<3$, and $2 \notin\{0,3\}$, we can conclude that we have an $l$-nps other than the type described above. In $\$ 4$ we shall show that the description of Theorem 1 does characterize $l$-nearest point sets in complete vector lattices. First we will investigate some further properties of members of $I$.

3. Decompositions of members of $I$. It was pointed out in the last section that $C$-sets could be written as the sum of a set of the form $A+(-A)$ and a normal subspace. This corresponds to demonstrating that each element of $I$ can be decomposed into a function whose image is a complex, and an additive member of $I$, because it is clear from Lemma 5 that if $A$ is a $C$-set, then $A \cap P$ defines a member of $I, \alpha$, whose image is $A \cap P$. Since $A=A \cap$ $P+(-A) \cap P$, by applying $\left(1^{\prime}\right)$, we have $A=[-\alpha, \alpha]$. The decomposition is achieved below.

THEOREM 2. If $\alpha \in I$, then there is $\alpha^{\prime}, \alpha^{*} \in I$ such that $\alpha^{\prime} \wedge \alpha^{*}=0, \alpha^{\prime}$ is additive, $\alpha^{*}(P)$ is a complex and $\alpha=\alpha^{*}+\alpha^{\prime}$.

Proof. Define $\alpha^{\prime}(x)=\sup \{\mu(x) \mid \mu \in I, \mu$ is additive and $\mu \leqslant \alpha\}$, for $x \in P$. It is clear that $\alpha^{\prime}: P \rightarrow P, \alpha^{\prime} \subseteq \geqslant, \alpha^{\prime}$ is isotone and $\alpha^{\prime}$ is idempotent (the latter since $\mu\left(\alpha^{\prime}(x)\right)=\mu(x) \wedge \alpha^{\prime}(x)=\mu(x)$, for $\mu$ such that $\mu(x) \leqslant$ $\alpha^{\prime}(x)$, by Lemma 4). A member $\gamma$ of $I$ is additive iff $\gamma(P)+(-\gamma(P))$ is a normal subgroup of $G$; i.e., iff $\gamma$ is a projection operator. Referring to the property $(x-\gamma(x)) \wedge \gamma(x)=0$, for $x \in P$, then if each of $\mu$ and $\beta$ are additive and $\leqslant \alpha$, then

$$
\begin{aligned}
(\mu+\beta)(x) & =\mu(x)+\beta(x-\mu(x))=\mu(x) \bigvee \beta(x-\mu(x)) \\
& \leqslant \alpha(x) \bigvee \alpha(x-\mu(x))=\alpha(x) .
\end{aligned}
$$

The latter inequality implies $\alpha^{\prime}$ is additive since it yields $\alpha^{\prime}(x)+\alpha^{\prime}(y) \leqslant \alpha^{\prime}(x$ $+y)$. Thus $\alpha^{\prime}$ is an additive member of $I$. Let $\alpha^{*}(x)=\alpha(x)-\alpha^{\prime}(x)$, for $x \in P$. If $0 \leqslant x \leqslant y$, then

$$
\begin{aligned}
\alpha^{\prime}(y) & =\alpha^{\prime}(\alpha(y))=\alpha^{\prime}(\alpha(y)-\alpha(x))+\alpha^{\prime}(\alpha(x)) \\
& \leqslant \alpha(y)-\alpha(x)+\alpha^{\prime}(x),
\end{aligned}
$$

which implies $\alpha^{*}$ is isotone. Since the other properties of members of $I$ are clearly possessed by $\alpha^{*}$, then $\alpha^{*}$ is in $I$. For $x \in P$,

$$
\begin{aligned}
\left(\alpha^{*} \wedge \alpha^{\prime}\right)(x) & =\left(\alpha(x)-\alpha^{\prime}(x)\right) \wedge \alpha^{\prime}(x) \\
& \leqslant\left(x-\alpha^{\prime}(x)\right) \wedge \alpha^{\prime}(x)=0,
\end{aligned}
$$

and we have $\alpha^{*} \wedge \alpha^{\prime}=0$. If $x>0$, then $n x \in \alpha^{*}(P)$ for every positive integer $n$ implies $p_{x} \leqslant \alpha^{*} \leqslant \alpha$, which leads, by definition of $\alpha^{\prime}$, to $p_{x} \leqslant \alpha^{\prime}$. The last statement is a contradiction. Therefore, for $x>0$, there is a positive integer $n$ such that $n x \notin \alpha^{*}(P)$; i.e., $\alpha^{*}(P)$ is a complex.

For $\alpha, \beta \in I$ and $x, y \in P$,

$$
\beta(y) \geqslant \beta(y)-(\alpha(\alpha(x)+\beta(y))-\alpha(x)),
$$


and, by Lemma 3,

$$
\begin{aligned}
(\alpha+\beta)(\alpha(x)+\beta(y))= & \alpha(\alpha(x)+\beta(y)) \\
& +(\alpha(x)+\beta(y)-\alpha(\alpha(x)+\beta(y))) \\
= & \alpha(x)+\beta(y),
\end{aligned}
$$

from which it follows that $\alpha(P)+\beta(P) \subseteq(\alpha+\beta)(P)$. The reverse containment is immediate, and we have $(\alpha+\beta)(P)=\alpha(P)+\beta(P)$. Pinsker $[6, \mathrm{pp} .65-67]$ proved that the collection of complexes with set addition as the operation is isomorphic to the positive cone of a complete l-group, and hence one in which $P$ can be embedded; $x \rightarrow \tau_{x}(P)$ is easily seen to be a morphism (for $z \in P$,

$$
\begin{aligned}
\left(\tau_{x}+\tau_{y}\right)(z) & =\tau_{x}(z)+\tau_{y}\left(z-\tau_{x}(z)\right) \\
& =x \wedge z+y \wedge(z-x \wedge z)=(x \wedge z+y) \wedge z \\
& =[(x+y) \wedge(z+y)] \wedge z \\
& \left.=(x+y) \wedge z=\tau_{x+y}(z)\right)
\end{aligned}
$$

The latter will be useful in Theorem 3 .

This theorem demonstrates that each complex can be decomposed as the direct sum of a collection of disjoint intervals of the form $[0, a]$. The decomposition suggests a different description of the positive cone $P^{*}$ of $G^{*}$ as a collection of equivalence classes containing disjoint subsets of $P$.

TheOREM 3. Let $\alpha \in I$ such that $\alpha(P)$ is a complex. There is a disjoint subset $A$ of $P$ such that $\alpha(x)=\sup \{x \wedge a \mid a \in A\}$, for $x \in P$.

Proof. Define $\beta(x)=\sup \left\{p_{z-\alpha(z)}(x) \mid z \in P\right\}$, for $x \in P$. As in the proof of Theorem 2, $\beta$ is an additive member of $I$.

$$
(\alpha+\beta)(x)=\alpha(x)+\beta(x-\alpha(x))=\alpha(x)+x-\alpha(x)=x,
$$

for $x \in P$. Since $x>0$ implies there is a positive integer $n$ such that $n x \neq \alpha(n x)$, then the above implies

$$
0<\beta(n x-\alpha(n x)) \leqslant \beta(n x)=n \beta(x) .
$$

Thus, $x>0$ leads to $\beta(x)>0$. Since $\beta(x-\beta(x))=0$, for $x \in P$, we have $\beta(x)=x$, for $x \in P$. A straightforward application of Kuratowski's Lemma will provide a disjoint subset $E$ of $P$ with each $e$ in $E \leqslant x_{e}-\alpha\left(x_{e}\right)$, for some $x_{e} \in P$, and $\sup \left\{p_{e}(x) \mid e \in E\right\}=x$, for $x \in P$. Let $\bar{e}=x_{e}-\alpha\left(x_{e}\right)$, for $e \in$ $E$.

$$
\begin{aligned}
\left(\alpha \wedge \tau_{\bar{e}}\right)(z) & =\alpha(z) \wedge \tau_{\bar{e}}(z)=\alpha(z) \wedge \bar{e} \\
& =z \wedge \alpha(\bar{e})=\tau_{\alpha(\bar{e})}(z) \leqslant \tau_{\alpha\left(x_{e}\right)}(z)
\end{aligned}
$$

for $z \in P$, by application of Lemma 4. If $n$ is a positive integer such that $\alpha \wedge n \tau_{\bar{e}}<\tau_{\alpha\left(x_{\bar{e}}\right)}$, where $n \tau_{\bar{e}}$ is defined to be $\tau_{\bar{e}}+\tau_{\bar{e}}+\cdots+\tau_{\bar{e}}$, then 


$$
\begin{aligned}
\alpha \wedge(n+1) \tau_{\bar{e}} & <\alpha \wedge\left(\alpha \wedge \tau_{\bar{e}}+\alpha \wedge n \tau_{\bar{e}}\right) \\
& <\alpha \wedge\left(\tau_{\bar{e}}+\tau_{\left.\alpha\left(x_{e}\right)\right)}=\alpha \wedge \tau_{\left(\bar{e}+\alpha\left(x_{e}\right)\right)}=\tau_{\alpha\left(x_{e}\right)},\right.
\end{aligned}
$$

and by induction we have $\alpha \wedge \tau_{\bar{e}}=\alpha \wedge n \tau_{\bar{e}} \leqslant \tau_{\alpha\left(x_{e}\right)}$, for every positive integer $n$, from which it follows that $\alpha \wedge p_{\bar{e}} \leqslant \tau_{\alpha\left(x_{e}\right)}$. Let $a_{e}=\left(\alpha \wedge p_{e}\right)\left(x_{e}\right)$, for $e \in E$; then $A=\left\{a_{e} \mid e \in E\right\}$ forms a disjoint subset of $P$ such that

$$
\begin{aligned}
\sup \{ & x \wedge a \mid a \in A\}=\sup \left\{x \wedge\left(\alpha \wedge p_{e}\right)\left(x_{e}\right) \mid e \in E\right\} \\
& =\sup \left\{\left(\alpha \wedge p_{e}\right)(x) \wedge x_{e} \mid e \in E\right\}=\sup \left\{p_{e}(x) \wedge \alpha(x) \wedge \alpha\left(x_{e}\right) \mid e \in E\right\} \\
& =\sup \left\{p_{e}(x) \wedge \alpha(x) \mid e \in E\right\}=\sup \left\{p_{e}(\alpha(x)) \mid e \in E\right\}=\alpha(x) .
\end{aligned}
$$

4. $l$-nearest point sets in a complete vector lattice. In this section we shall return to a study of $l$-nearest point sets. In order to arrive at the theorem characterizing $l$-nearest point sets, we must first prove technical lemmas about such sets. Again $G$ is taken to be a complete $l$-group.

LEMMA 6. Let $S$ be an l-nps; then for $x \in G, f_{S}\left(x \vee f_{S}(x)\right)=f_{S}(x)$.

Proof.

$$
\begin{aligned}
\left|x \vee f_{S}(x)-f_{S}(x)\right| & =\left(x-f_{S}(x)\right) \vee 0 \\
& =\left|x-f_{S}(x)\right|+\left(x-f_{S}(x)\right) \wedge 0 \\
& \leqslant\left|x-f_{S}\left(x \vee f_{S}(x)\right)\right|-\left(f_{S}(x)-x\right) \vee 0 \\
& =|| x-f_{S}\left(x \vee f_{S}(x)\right)|-|\left(f_{S}(x)-x\right) \vee 0|| \\
& \leqslant\left|x-f_{S}\left(x \vee f_{S}(x)\right)+\left(f_{S}(x)-x\right) \vee 0\right| \\
& =\left|x \vee f_{S}(x)-f_{S}\left(x \vee f_{S}(x)\right)\right|,
\end{aligned}
$$

by applying properties of complete $l$-groups along with Lemma 1 (iii).

Lemma 7. Let $S$ be an l-nps, $\{0, t\} \subseteq S \cap P$ and $m \in[0, t]$ such that $m \wedge(t-m)=0$; then $m \in S$.

Proof. $\left|m-f_{S}(m)\right| \leqslant|m-0| \wedge|m-t|=m \wedge(t-m)=0$.

In the remainder of the paper $G$ will be taken to be a complete vector lattice (see [3] for definitions). The main difficulty in demonstrating the converse of Theorem 1 is, as the example of $\$ 2$ suggests, in establishing the convexity of an $l$-nps. The lemma below provides an essential tool for doing so.

Lemma 8. If $S$ is an l-nps, $\{0, t\} \subseteq S \cap P$ and $x \in[0, t]$ such that $f_{S}(x)=$ 0 , then $x=0$.

Proof. Since $x=\left|x-f_{S}(x)\right|<|x-t|=t-x$, we have

$$
T=\{y \mid 2 x \leqslant y \text { and } y \in S \cap[0, t]\} \neq \Phi,
$$

and thus $w=\inf T$ exists and is in $S \cap[0, t]$. 


$$
w / 2-f_{S}(w / 2) \leqslant\left|w / 2-f_{S}(w / 2)\right| \leqslant|w / 2-0|=w / 2,
$$

implies $f_{S}(w / 2) \geqslant 0$. Let $r=\left(x-f_{S}(w / 2)\right)^{+}$, and note that $p_{r}\left(f_{S}(w / 2)\right) \in S$ by Lemma 7. Since $r \wedge\left(x-f_{S}(w / 2)\right)^{-}=0$,

$$
\begin{aligned}
0 & =p_{r}\left(f_{S}(w / 2)\right) \wedge\left(\left(f_{S}(w / 2)-x\right) \vee 0\right) \\
& =\left[p_{r}\left(f_{S}(w / 2)\right) \wedge\left(f_{S}(w / 2)-x\right)\right] \vee\left[p_{r}\left(f_{S}(w / 2)\right) \wedge 0\right] \\
& =\left(\left[\left(p_{r}\left(f_{S}(w / 2)\right)+x\right) \wedge f_{S}(w / 2)\right]-x\right) \vee 0,
\end{aligned}
$$

from which it follows that

$$
p_{r}\left(f_{S}(w / 2)\right) \leqslant\left(p_{r}\left(f_{S}(w / 2)\right)+x\right) \wedge f_{S}(w / 2) \leqslant x .
$$

Thus

$$
\left|x-p_{r}\left(f_{S}(w / 2)\right)\right| \leqslant x=\left|x-f_{S}(x)\right|,
$$

and we have $p_{r}\left(f_{S}(w / 2)\right)=0$. Since

$$
0 \leqslant p_{r}(w) \wedge f_{S}(w / 2)=w \wedge p_{r}\left(f_{S}(w / 2)\right)=0,
$$

by Lemma 4 , then we can apply Lemma 1(i), (ii), and note that

$$
\begin{aligned}
\mid w / 2- & p_{r}(w)-f_{S}(w / 2) \mid \\
& =\left|\left(w / 2-p_{r}(w / 2)-f_{S}(w / 2)\right)-p_{r}(w / 2)\right| \\
& =\left|w / 2-p_{r}(w / 2)-f_{S}(w / 2)+p_{r}(w / 2)\right|=\left|w / 2-f_{S}(w / 2)\right| .
\end{aligned}
$$

The fact that $p_{r}(w)+f_{S}(w / 2)=p_{r}(w) \vee f_{S}(w / 2) \in S$ implies that $f_{S}(w / 2)$ $=p_{r}(w)+f_{S}(w / 2)$, and hence $p_{r}(w)=0$. But $0 \leqslant r \leqslant x \leqslant w$ implies $r=$ $p_{r}(r)=0$, and hence $x-f_{S}(w / 2) \leqslant 0$. Therefore $x=\left|x-f_{S}(w / 2)\right|=$ $f_{S}(w / 2)-x$, and we have that $f_{S}(w / 2) \in T$, from which it follows that $w \leqslant f_{S}(w / 2)$. The latter tells us that

$$
\begin{aligned}
\left|w / 2-f_{S}(w / 2)\right| & =f_{S}(w / 2)-w / 2>w-w / 2 \\
& =|w / 2-w|=|w / 2-0|,
\end{aligned}
$$

from which we may conclude $0=f_{S}(w / 2)=w$. The result follows.

With the lemma above we can proceed to prove the convexity of an $l$-nps $S$ as follows: Suppose $a, b \in S$ and $a \leqslant b$. Then $x$ in $[a, b]$ implies that $f_{S}(x) \leqslant x \vee f_{S}(x) \leqslant b \vee f_{S}(x) \in S$. Define $T=S-f_{S}(x)$; then $T$ is an $l$-nps, $t=b \vee f_{S}(x)-f_{S}(x) \in T$, and $x \vee f_{S}(x)-f_{S}(x) \in[0, t]$ such that $f_{T}\left(x \vee f_{S}(x)-f_{S}(x)\right)=0$. By Lemma $8, x \vee f_{S}(x)-f_{S}(x)=0$, or $f_{S}(x)>$ $x$. Since $-S$ is an $l$-nps, with $-b \leqslant-x \leqslant-a$, the same argument produces $f_{-s}(-x) \geqslant-x$; i.e., $f_{S}(x) \leqslant x$. Thus we have proven the convexity of $S$.

LEMMA 9. If $S$ is an l-nps and $a, b \in S$ such that $a \leqslant b$, then $[a, b] \subseteq S$.

We can now demonstrate the converse to Theorem 1 .

TheOREM 4. Let $G$ be a complete vector lattice. $S$ is an l-nps iff $S=x+$ $[-\alpha, \beta]$, for some $x \in G$ and $\alpha, \beta \in I$. 
Proof. Suppose $S$ is an $l$-nps and $x \in S$. Then $T=S-x$ is an $l$-nps and $0 \in T$. Let $\beta(x)=\sup \{x \wedge y \mid y \in T\}$, and $\alpha(x)=\sup \{x \wedge y \mid y \in-T\}$, for $x \in P$. It is clear that $\alpha, \beta \in I$, by Lemma 5 , and since $x \wedge y \in[0, y]$, for $y \in T \cap P$, then $x \wedge y \in T \cap P$, by Lemma 9, and we have $\beta(x)=$ $\sup \{y \mid y \in T$ and $y \leqslant x\}$, which is in $T$ by Lemma 2 . Similarly for $\alpha$. Thus $[0, \beta] \subseteq T \cap P$ and $[-\alpha, 0] \subseteq T \cap(-P)$. Since the reverse containment is clear, we have

$$
[-\alpha, \beta]=[-\alpha, 0]+[0, \beta]=T \cap(-P)+T \cap P=T .
$$

The converse implication follows from Theorem 1 .

\section{BIBLIOGRAPHY}

1. W. D. L. Appling, Set functions and an extremal problem in absolute continuity, Rend. Circ. Mat. Palermo (2) 14 (1965), 256-266.

2. A A generalization of absolute continuity and of an analogue of the Lebesgue decomposition theorem, Riv. Mat. Univ. Parma (3) 2 (1973), 251-276.

3. G. Birkhoff, Lattice theory, Amer. Math. Soc. Colloq. Publ., vol. 25, Amer. Math. Soc., Providence, R. I., 1967.

4. S. Bochner and R. S. Phillips, Additive set functions and vector lattices, Ann. of Math. (2) 42 (1941), 316-324.

5. S. Kakutani, Concrete representation of abstract (L)-spaces and the mean ergodic theorem, Ann. of Math. (2) 42 (1941), 523-537.

6. L. V. Kantorovič, B. Z. Vulih and A. G. Pinsker, Partially ordered groups and partially ordered linear spaces, Amer. Math. Soc. Transl. (2) 27 (1963), 51-124.

7. A. G. Pinsker, On the extension of partially ordered spaces, Dokl. Akad. Nauk SSSR 21 (1938), 6-10. (Russian)

8. , On some properties of extended K-spaces, Dokl. Akad. Nauk SSSR 22 (1939), 220-224. (Russian)

Department of Mathematics, Arkansas Tech University, RussellVille, Arkansas 72801 